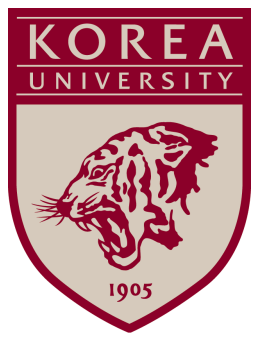

Discussion Paper Series

No. 1501

May 2015 (updated)

\title{
On Budget Balance of the Dynamic Pivot Mechanism
}

\author{
Kiho Yoon
}




\title{
On Budget Balance of the Dynamic Pivot Mechanism
}

\author{
Kiho Yoon \\ Department of Economics, Korea University \\ 145 Anam-ro, Seongbuk-gu, Seoul, Korea 136-701 \\ kiho@korea.ac.kr \\ http://econ.korea.ac.kr/ ${ }^{\sim}$ kiho
}

This Version: May 2015

First Version: February 2014

\begin{abstract}
We modify the dynamic pivot mechanism of Bergemann and Välimäki (Econometrica, 2010) in such a way that lump-sum fees are collected from the players. We show that the modified mechanism satisfies ex-ante budget balance as well as ex-post efficiency, periodic ex-post incentive compatibility, and periodic ex-post individual rationality, as long as the Markov chain representing the evolution of players' private information is irreducible and aperiodic and players are sufficiently patient. We also show that the diverse preference assumption of Bergemann and Välimäki may preclude budget balance.
\end{abstract}

JEL Classification: C73, D47, D82

Keywords: The dynamic pivot mechanism, dynamic mechanism design, budget balance, VCG mechanism, bilateral trading 


\section{On Budget Balance of the Dynamic Pivot Mechanism}

\section{Introduction}

The research on dynamic mechanism design is recently surging. As the surveys of Bergemann and Said (2010) and Vohra (2012) suggest, the literature can be divided into two categories. The first category deals with the environments in which the population of players changes over time, but their private information is fixed, whereas the second category deals with the environments in which the population of players is fixed, but their private information changes over time.

This paper belongs to the second category. Notable papers in this category include Athey and Segal (2013) and Pavan, Segal, and Toikka (2014). In particular, Bergemann and Välimäki (2010) introduced the dynamic pivot mechanism, which is a generalization of the renowned VCG (Vickrey-Clarke-Groves) mechanism to the dynamic setting. The dynamic pivot mechanism is ex-post efficient, periodic ex-post incentive compatible, and periodic ex-post individually rational. The problem with this mechanism, however, is that it may run an expected budget deficit. In this paper, we modify the dynamic pivot mechanism in a way that lump-sum fees are collected from the players so that the modified mechanism satisfies ex-ante budget balance as well as the aforementioned property of expost efficiency, periodic ex-post incentive compatibility, and periodic ex-post individual rationality.

In the next section, we set up a general model and establish that the dynamic pivot mechanism with lump-sum fees is ex-post efficient, periodic ex-post incentive compatible and individually rational, and ex-ante budget balancing if the Markov chain representing the evolution of players' private information is irreducible and aperiodic and players are sufficiently patient. This result holds essentially since the participation constraints of the worst-off types are relaxed in the dynamic setting. On the other hand, we show that the diverse preference assumption of Bergemann and Välimäki (2010) may preclude budget 
balance. The reason is that this assumption makes the Markov chain reducible. Section 3 contains discussion on recent related research and future agenda.

\section{Main Results}

We first present a general model based on Bergemann and Välimäki (2010), and then discuss budget balance of the dynamic pivot mechanism.

\subsection{The setup}

There is a set $I=\{1, \ldots, n\}$ of players and a countable number of periods, indexed by $t \in\{0,1, \ldots\}$. Player $i$ 's type in period $t$ is $\theta_{i}^{t} \in \Theta_{i}$. We assume that this is private information. Let $\theta^{t}=\left(\theta_{1}^{t}, \ldots, \theta_{n}^{t}\right)$ and $\Theta=\prod_{i=1}^{n} \Theta_{i} \cdot{ }^{1}$ To deliver the main idea without getting involved into many subtle mathematical issues, we will assume that $\Theta$ is discrete (i.e., finite or countably infinite). After $\theta^{t} \in \Theta$ is realized in period $t$, a public action $a^{t} \in A$ is determined. In addition, let $z_{i}^{t} \in \mathbb{R}$ be a monetary transfer from player $i$ in period $t$. Given sequences $\left(\theta^{0}, \theta^{1}, \ldots\right)$ of type profiles and $\left(a^{0}, a^{1}, \ldots\right)$ of actions, together with $\left(z_{i}^{0}, z_{i}^{1}, \ldots\right)$ of $i$ 's monetary transfers, player $i$ 's payoff is

$$
\sum_{t=0}^{\infty} \delta^{t}\left(v_{i}\left(\theta_{i}^{t}, a^{t}\right)-z_{i}^{t}\right)
$$

where (i) $\delta$ is a common discount factor and $\delta<1$, and (ii) $v_{i}(\cdot)$ is a reward function. Note that we deal with the private-values environment in that player $i$ 's reward function depends only on player $i$ 's type. We also assume that $\left|v_{i}\left(\theta_{i}, a\right)\right| \leq C<\infty$ for all $i, \theta_{i}$, and $a$.

The dynamic evolution of players' types is represented by a Markov chain. Let $p\left(\theta^{t+1} \mid \theta^{t}, a^{t}\right)$ be the transition probability that type profile $\theta^{t+1}$ will be realized in period $t+1$ when the type profile is $\theta^{t}$ and the action is $a^{t}$ in period $t$. Observe that, except for the fact that $\theta$ is private information, this environment fits into a Markov decision

1 We may include public information, say $\theta_{0}^{t} \in \Theta_{0}$, to be more realistic. We dispense with this additional notation for clearer presentation of the main idea. 
process with $\Theta$ being the set of states. ${ }^{2}$

We consider a dynamic direct mechanism that asks each player to report her type (i.e., state) in each period. Let $r_{i}^{t}$ denote player $i$ 's report in period $t$, which may or may not be equal to her true type $\theta_{i}^{t}$. Let

$$
h_{i}^{t}=\left(\theta_{i}^{0}, r^{0}, a^{0}, \theta_{i}^{1}, r^{1}, a^{1}, \ldots, \theta_{i}^{t-1}, r^{t-1}, a^{t-1}, \theta_{i}^{t}\right)
$$

be a private history of player $i$ in period $t$, where each $r^{s}=\left(r_{1}^{s}, \ldots, r_{n}^{s}\right)$ for $s=0, \ldots, t-1$ is a report profile, and let $H_{i}^{t}$ be the set of all such histories. A (pure) strategy for player $i$ in period $t$ is a function $\hat{r}_{i}^{t}: H_{i}^{t} \rightarrow \Theta_{i}$. A strategy is truth-telling if $\hat{r}_{i}^{t}\left(h_{i}^{t}\right)=\theta_{i}^{t}$ for all $h_{i}^{t}$. In addition, let

$$
h^{t}=\left(r^{0}, a^{0}, r^{1}, a^{1}, \ldots, r^{t-1}, a^{t-1}, r^{t}\right)
$$

be a public history in period $t$ and let $H^{t}$ be the set of all such histories. Observe that, when players adopt the truth-telling strategy, the private histories do not contain more information than the public histories on the equilibrium path. Since we are mainly concerned with incentive compatible mechanisms in which the truth-telling strategy is an equilibrium, we will not henceforth distinguish between true states and reported states (mainly to save notations).

In each period, the mechanism decides on the action based on the actions chosen up to the previous period and the reports up to the beginning of this period. Thus, when players adopt the truth-telling strategy, a deterministic (history-dependent) decision rule of the mechanism in period $t$ is a function $\hat{a}^{t}: H^{t} \rightarrow A$. A special class of decision rule is the deterministic Markovian decision rule that chooses an action based only on the current state, i.e., $\hat{a}^{t}: \Theta \rightarrow A$. Moreover, a randomized decision rule $\hat{a}^{t}$ specifies a probability distribution on the set of actions. Randomized decision rules may be history-dependent or Markovian. A policy of the mechanism is a sequence of decision rules $\pi=\left(\hat{a}^{0}, \hat{a}^{1}, \ldots\right)$. Let $\Pi$ be the set of all policies.

2 Note that this Markov formulation is essentially without loss of generality, since any dynamic model can be described using Markov notation by expanding the state space appropriately. See Stokey and Lucas (1989) or Athey and Segal (2013) for an excellent discussion. 
An efficient policy is $\pi^{*} \in \Pi$ that maximizes the expected discounted sum of players' rewards. That is,

$$
\pi^{*} \in \arg \max _{\pi \in \Pi} E_{\theta}^{\pi}\left[\sum_{t=0}^{\infty} \delta^{t} \sum_{i=1}^{n} v_{i}\left(\tilde{\theta}_{i}^{t}, \tilde{a}^{t}\right)\right]
$$

for every $\theta \in \Theta$. By the way, we will assume throughout that the relevant maximum is attained without specifying sufficient conditions. Since $\Theta$ is discrete, this assumption is valid when (i) $A$ is finite or (ii) $A$ is compact, $v_{i}\left(\theta_{i}, a\right)$ is continuous in $a$ for each $i$ and $\theta_{i}$, and $p\left(\theta^{\prime} \mid \theta, a\right)$ is continuous in $a$ for each $\theta$ and $\theta^{\prime}$. Other sufficient conditions may also guarantee the existence of an efficient policy $\pi^{*}{ }^{3}$

In addition, the mechanism specifies the monetary transfers based on public histories. A deterministic (history-dependent) transfer rule of the mechanism in period $t$ is a collection of functions $\left\{\hat{z}_{i}^{t}: H^{t} \rightarrow \mathbb{R}\right\}_{i \in I}$. Let $\hat{z}^{t}=\left(\hat{z}_{1}^{t}, \ldots, \hat{z}_{n}^{t}\right)$. Markovian transfer rules and randomized transfer rules can be defined similarly. We assume that the mechanism is committed to future transfers. In summary, a dynamic direct mechanism is represented by a family of decision rules and monetary transfer rules, $\left\{\hat{a}^{t}, \hat{z}^{t}\right\}_{t=0}^{\infty}$.

We call a policy stationary if $\hat{a}^{t}=\hat{a}$ for all $t$. A stationary policy has the form $\pi=(\hat{a}, \hat{a}, \ldots)$, which is denoted by $\hat{a}^{\infty}$. For the stationary environment considered in this paper, we can restrict our attention to deterministic stationary policies when finding a policy that maximizes the expected discounted sum of players' rewards. ${ }^{4}$ An efficient policy thus has the form $\pi^{*}=\left(a^{*}\right)^{\infty}$ where $a^{*}: \Theta \rightarrow A$.

The social welfare function $W: \Theta \rightarrow \mathbb{R}$ can be defined recursively by the following optimality equation (or Bellman equation):

$$
W(\theta)=\sum_{i=1}^{n} v_{i}\left(\theta_{i}, a^{*}(\theta)\right)+\delta \sum_{\theta^{\prime} \in \Theta} p\left(\theta^{\prime} \mid \theta, a^{*}(\theta)\right) W\left(\theta^{\prime}\right) .
$$

By the way, with the normalization of players' autarky payoff levels to zero, it is natural to impose the positive surplus assumption that $\sum_{i=1}^{n} v_{i}\left(\theta_{i}, a^{*}(\theta)\right) \geq 0$ for all $\theta \in \Theta$, with at

3 See Proposition 4.4.3. of Puterman (2005) or Stokey and Lucas (1989).

4 See Theorem 6.2.7. of Puternam (2005). Note that a deterministic stationary policy is a deterministic Markovian policy. 
least one strict inequality. The weak inequality holds since one alternative available to the mechanism is to let the players enjoy their autarky payoff levels, and the strict inequality is assumed since, otherwise, the problem is not interesting.

\subsection{The dynamic pivot mechanism}

To define the dynamic pivot mechanism, we use the usual notational convention that the subscript $-i$ pertains to players other than $i$. Thus, $\theta_{-i}=\left(\theta_{1}, \ldots, \theta_{i-1}, \theta_{i+1}, \ldots, \theta_{n}\right)$, $\Theta_{-i}=\prod_{j \neq i} \Theta_{j}$, and so on. Let $\pi_{-i}^{*}=\left(a_{-i}^{*}\right)^{\infty}$ where $a_{-i}^{*}: \Theta \rightarrow A$ is a deterministic decision rule that maximizes the expected discounted sum

$$
E_{\theta}^{\pi}\left[\sum_{t=0}^{\infty} \delta^{t} \sum_{j \neq i} v_{j}\left(\tilde{\theta}_{j}^{t}, \tilde{a}^{t}\right)\right]
$$

of the rewards of players other than $i .^{5}$ Define the social welfare for players other than $i$, denoted by $W_{-i}: \Theta \rightarrow \mathbb{R}$, recursively by

$$
W_{-i}(\theta)=\sum_{j \neq i} v_{j}\left(\theta_{j}, a_{-i}^{*}(\theta)\right)+\delta \sum_{\theta^{\prime} \in \Theta} p\left(\theta^{\prime} \mid \theta, a_{-i}^{*}(\theta)\right) W_{-i}\left(\theta^{\prime}\right)
$$

Define player $i$ 's marginal contribution $M_{i}(\theta)=W(\theta)-W_{-i}(\theta)$, and in addition, the flow marginal contribution $m_{i}(\theta)$ by the equation

$$
M_{i}(\theta)=m_{i}(\theta)+\delta \sum_{\theta^{\prime} \in \Theta} p\left(\theta^{\prime} \mid \theta, a^{*}(\theta)\right) M_{i}\left(\theta^{\prime}\right)
$$

Then,

$$
\begin{aligned}
m_{i}(\theta) & =W(\theta)-W_{-i}(\theta)-\delta \sum_{\theta^{\prime} \in \Theta} p\left(\theta^{\prime} \mid \theta, a^{*}(\theta)\right)\left(W\left(\theta^{\prime}\right)-W_{-i}\left(\theta^{\prime}\right)\right) \\
& =\sum_{j=1}^{n} v_{j}\left(\theta_{j}, a^{*}(\theta)\right)-\sum_{j \neq i} v_{j}\left(\theta_{j}, a_{-i}^{*}(\theta)\right) \\
& +\delta\left(\sum_{\theta^{\prime} \in \Theta} p\left(\theta^{\prime} \mid \theta, a^{*}(\theta)\right) W_{-i}\left(\theta^{\prime}\right)-\sum_{\theta^{\prime} \in \Theta} p\left(\theta^{\prime} \mid \theta, a_{-i}^{*}(\theta)\right) W_{-i}\left(\theta^{\prime}\right)\right) .
\end{aligned}
$$

\footnotetext{
5 The existence of such a deterministic stationary policy is guaranteed by our assumptions.
} 
The dynamic pivot mechanism is a dynamic direct mechanism with an efficient policy $\pi^{*}=\left(a^{*}\right)^{\infty}$ together with the stationary monetary transfer rule $z_{i}^{*}: \Theta \rightarrow \mathbb{R}$ given as

$$
\begin{aligned}
z_{i}^{*}(\theta) & =v_{i}\left(\theta_{i}, a^{*}(\theta)\right)-m_{i}(\theta) \\
& =\sum_{j \neq i}\left(v_{j}\left(\theta_{j}, a_{-i}^{*}(\theta)\right)-v_{j}\left(\theta_{j}, a^{*}(\theta)\right)\right) \\
& +\delta\left(\sum_{\theta^{\prime} \in \Theta} p\left(\theta^{\prime} \mid \theta, a_{-i}^{*}(\theta)\right) W_{-i}\left(\theta^{\prime}\right)-\sum_{\theta^{\prime} \in \Theta} p\left(\theta^{\prime} \mid \theta, a^{*}(\theta)\right) W_{-i}\left(\theta^{\prime}\right)\right) .
\end{aligned}
$$

The notion of incentive compatibility is the periodic ex-post incentive compatibility: A dynamic direct mechanism is periodic ex-post incentive compatible if the truth-telling strategy is a best response for every player $i$ and every true type profile $\theta$ in every period t. Bergemann and Välimäki (2010) have shown in their theorem 1 that the dynamic pivot mechanism is periodic ex-post incentive compatible.

Observe that player $i$ 's flow payoff in the dynamic pivot mechanism when the type profile is $\theta$ is $m_{i}(\theta)$. Observe also that player $i$ 's payoff in a period with type profile $\theta$ is $M_{i}(\theta)$, which is non-negative by definition of $W(\theta)$ and $W_{-i}(\theta)$ together with the normalization of autarky payoff levels to zero. Thus, the periodic ex-post participation constraints are satisfied. We note that, whereas commitment of the mechanism to future transfers is assumed, no commitment of the players is necessary for the dynamic pivot mechanism since the mechanism is periodic ex-post incentive compatible and individually rational.

It is well-known that the dynamic pivot mechanism may run a budget deficit even in expectation for many problems, such as the dynamic bilateral trading. To cope with the budget problem, we modify the dynamic pivot mechanism in a way that lump-sum (participation) fees are collected from the players. ${ }^{6}$

6 The idea of charging lump-sum fees in the class of Groves mechanisms appears in Makowski and Mezzetti (1994), Williams (1999), Krishna and Perry (2000), and Yoon (2001, 2008) for the static setting as well as in Skrzypacz and Toikka (2015) for the dynamic setting. 


\subsection{Budget balance of the dynamic pivot mechanism}

We now introduce several notations to discuss budget balance. Let $x \in \Delta(\Theta)$ denote the initial distribution of $\theta^{0}=\left(\theta_{1}^{0}, \ldots, \theta_{n}^{0}\right)$, and let $P$ be the transition matrix on $\Theta$. Thus, we assume that the transition probability is independent of the action $a$. Then, the distribution of $\theta^{t}$ is $x^{T} P^{t}$, where $x^{T}$ is the transpose of the column vector $x$ and $P^{t}$ is the $t$-th power of the matrix $P$. The flow budget deficit when the state is $\theta$ is $-\sum_{i=1}^{n} z_{i}^{*}(\theta)$. Let $-z^{*}$ be the column vector of flow budget deficit, i.e., $-z^{*}=\left(-\sum_{i=1}^{n} z_{i}^{*}(\theta)\right)_{\theta \in \Theta}$. Then, the expected budget deficit in period $t$ is given by $-x^{T} P^{t} z^{*}$. Let $x_{i \mid \theta_{i}^{0}}$ be the conditional distribution vector of initial distribution, given $\theta_{i}^{0}$. Since $i$ knows her type $\theta_{i}^{0}$ at the beginning of period zero, $x_{i \mid \theta_{i}^{0}}^{T} P^{t}$ is the distribution of $\theta^{t}$ that player $i$ with $\theta_{i}^{0}$ perceives. Let $m_{i}$ be the column vector of $i$ 's flow payoffs, i.e.,

$$
m_{i}=\left(v_{i}\left(\theta_{i}, a^{*}(\theta)\right)-z_{i}^{*}(\theta)\right)_{\theta \in \Theta} .
$$

Then, the expected payoff of player $i$ with $\theta_{i}^{0}$ in period $t$ is $x_{i \mid \theta_{i}^{0}} P^{t} m_{i}$. Thus, ex-ante budget balance can be achieved with lump-sum fees if and only if

$$
-\sum_{t=0}^{\infty} \delta^{t} x^{T} P^{t} z^{*} \leq \sum_{i=1}^{n} \inf _{\theta_{i}^{0} \in \Theta_{i}(x)} \sum_{t=0}^{\infty} \delta^{t} x_{i \mid \theta_{i}^{0}}^{T} P^{t} m_{i}
$$

where $\Theta_{i}(x)$ is the set of $\theta_{i} \in \Theta_{i}$ for which there exists $\theta_{-i}$ such that $\left(\theta_{i}, \theta_{-i}\right)$ belongs to the support of $x$. That is, ex-ante budget balance can be achieved if and only if the expected discounted sum of the payoffs of the worst-off types is greater than or equal to the expected budget deficit: The mechanism can charge sufficient lump-sum fees to cover the budget deficit without violating any player's participation constraint.

Observe that, since $M_{i}(\theta) \geq 0$ for all $i$ and $\theta$, the inequality $(*)$ is easily satisfied when $\sum_{t=0}^{\infty} \delta^{t} x^{T} P^{t} z^{*} \geq 0$, for example, when $z_{i}^{*}(\theta) \geq 0$ for all $i$ and $\theta$. Since each player's transfer is equal to her negative externality on the society in the VCG mechanism and consequently in the dynamic pivot mechanism, we have $z_{i}^{*}(\theta) \geq 0$ for environments with nonpositive externality such as the auction. In contrast, for environments with positive externality, such as the bilateral trading, the various double auctions in which some players 
are suppliers of the resources, or the situation in which multiple players are needed together to improve the social welfare, we generally have $\sum_{t=0}^{\infty} \delta^{t} x^{T} P^{t} z^{*}<0$. We now present a sufficiency result for ex-ante budget balance.

Theorem 1. Assume that the transition probability is independent of the action. Assume also that the set $\Theta$ is finite and the Markov chain is irreducible and aperiodic. Then, for any initial distribution $x \in \Delta(\Theta)$, ex-ante budget balance is achieved for sufficiently large $\delta$.

Proof. Let $v_{i}=\left(v_{i}\left(\theta_{i}, a^{*}(\theta)\right)\right)_{\theta \in \Theta}$. Then,

$$
-\sum_{t=0}^{\infty} \delta^{t} x^{T} P^{t} z^{*}=\sum_{i=1}^{n} \sum_{t=0}^{\infty} \delta^{t} x^{T} P^{t} m_{i}-\sum_{i=1}^{n} \sum_{t=0}^{\infty} \delta^{t} x^{T} P^{t} v_{i}
$$

and

$$
\sum_{i=1}^{n} \min _{\theta_{i}^{0} \in \Theta_{i}(x)} \sum_{t=0}^{\infty} \delta^{t} x_{i \mid \theta_{i}^{0}}^{T} P^{t} m_{i}=\sum_{i=1}^{n} \sum_{t=0}^{\infty} \delta^{t} \underline{x}_{i}^{T} P^{t} m_{i}
$$

where $\underline{x}_{i}$ is a value that attains the minimum for $i=1, \ldots, n$. Thus, $(*)$ is satisfied if and only if

$$
\sum_{i=1}^{n} \sum_{t=0}^{\infty} \delta^{t} \underline{x}_{i}^{T} P^{t} m_{i}-\sum_{i=1}^{n} \sum_{t=0}^{\infty} \delta^{t} x^{T} P^{t} m_{i}+\sum_{i=1}^{n} \sum_{t=0}^{\infty} \delta^{t} x^{T} P^{t} v_{i} \geq 0 .
$$

Let $p_{\theta \theta^{\prime}}^{(t)}$ be the $\theta \theta^{\prime}$-th element of $P^{t}$. By the well-known facts on finite Markov chains, there is a unique stationary distribution $\mu$ such that (i) $p_{\theta \theta^{\prime}}^{(t)} \rightarrow \mu_{\theta^{\prime}}$ as $t \rightarrow \infty$ for all $\theta, \theta^{\prime} \in \Theta$, and (ii) $\mu_{\theta^{\prime}}>0$ for all $\theta^{\prime} \in \Theta$. Thus, for any $\epsilon>0$, there is $t_{0}$ such that $\left|p_{\theta \theta^{\prime}}^{(t)}-\mu_{\theta^{\prime}}\right|<\epsilon$ for $t \geq t_{0}$.

Observe that, for $t \geq t_{0}$, we have

$$
\underline{x}_{i}^{T} P^{t} m_{i}=\sum_{\theta \in \Theta} \sum_{\theta^{\prime} \in \Theta} p_{\theta \theta^{\prime}}^{(t)} \underline{x}_{i, \theta} m_{i, \theta^{\prime}}>\sum_{\theta \in \Theta} \sum_{\theta^{\prime} \in \Theta}\left(\mu_{\theta^{\prime}}-\epsilon\right) \underline{x}_{i, \theta} m_{i, \theta^{\prime}}=\sum_{\theta^{\prime} \in \Theta}\left(\mu_{\theta^{\prime}}-\epsilon\right) m_{i, \theta^{\prime}}
$$

since $\sum_{\theta} \underline{x}_{i, \theta}=1$. (Here, $\underline{x}_{i, \theta}$ is the $\theta$-th element of the column vector $\underline{x}_{i}$ and $m_{i, \theta^{\prime}}$ is the $\theta^{\prime}$-th element of the column vector $m_{i}$. The same convention applies below.) Thus,

$$
\sum_{i=1}^{n} \sum_{t=0}^{\infty} \delta^{t} \underline{x}_{i}^{T} P^{t} m_{i}>\sum_{i=1}^{n} \sum_{t=0}^{t_{0}-1} \delta^{t} \underline{x}_{i}^{T} P^{t} m_{i}+\frac{\delta^{t_{0}}}{1-\delta} \sum_{\theta^{\prime} \in \Theta}\left(\mu_{\theta^{\prime}}-\epsilon\right)\left(\sum_{i=1}^{n} m_{i, \theta^{\prime}}\right) .
$$


Likewise,

$$
\sum_{i=1}^{n} \sum_{t=0}^{\infty} \delta^{t} x^{T} P^{t} m_{i}<\sum_{i=1}^{n} \sum_{t=0}^{t_{0}-1} \delta^{t} x^{T} P^{t} m_{i}+\frac{\delta^{t_{0}}}{1-\delta} \sum_{\theta^{\prime} \in \Theta}\left(\mu_{\theta^{\prime}}+\epsilon\right)\left(\sum_{i=1}^{n} m_{i, \theta^{\prime}}\right)
$$

and

$$
\sum_{i=1}^{n} \sum_{t=0}^{\infty} \delta^{t} x^{T} P^{t} v_{i}>\sum_{i=1}^{n} \sum_{t=0}^{t_{0}-1} \delta^{t} x^{T} P^{t} v_{i}+\frac{\delta^{t_{0}}}{1-\delta} \sum_{\theta^{\prime} \in \Theta}\left(\mu_{\theta^{\prime}}-\epsilon\right)\left(\sum_{i=1}^{n} v_{i, \theta^{\prime}}\right) .
$$

Therefore,

$$
\begin{aligned}
& \sum_{i=1}^{n} \sum_{t=0}^{\infty} \delta^{t} \underline{x}_{i}^{T} P^{t} m_{i}-\sum_{i=1}^{n} \sum_{t=0}^{\infty} \delta^{t} x^{T} P^{t} m_{i}+\sum_{i=1}^{n} \sum_{t=0}^{\infty} \delta^{t} x^{T} P^{t} v_{i} \\
> & \sum_{i=1}^{n} \sum_{t=0}^{t_{0}-1} \delta^{t} \underline{x}_{i}^{T} P^{t} m_{i}-\sum_{i=1}^{n} \sum_{t=0}^{t_{0}-1} \delta^{t} x^{T} P^{t} m_{i}+\sum_{i=1}^{n} \sum_{t=0}^{t_{0}-1} \delta^{t} x^{T} P^{t} v_{i} \\
+ & \frac{\delta^{t_{0}}}{1-\delta} \sum_{\theta^{\prime} \in \Theta}\left\{\left(\mu_{\theta^{\prime}}-\epsilon\right)\left(\sum_{i=1}^{n} v_{i, \theta^{\prime}}\right)-2 \epsilon\left(\sum_{i=1}^{n} m_{i, \theta^{\prime}}\right)\right\} .
\end{aligned}
$$

Now,

$$
\sum_{i=1}^{n} \sum_{t=0}^{t_{0}-1} \delta^{t} x^{T} P^{t} v_{i} \geq-\frac{1-\delta^{t_{0}}}{1-\delta} n C
$$

since $\left|v_{i}\left(\theta_{i}, a\right)\right| \leq C$ by assumption. Likewise,

$$
\sum_{i=1}^{n} \sum_{t=0}^{t_{0}-1} \delta^{t} \underline{x}_{i}^{T} P^{t} m_{i} \geq-\frac{1-\delta^{t_{0}}}{1-\delta} n C^{\prime} \text { and } \sum_{i=1}^{n} \sum_{t=0}^{t_{0}-1} \delta^{t} x^{T} P^{t} m_{i} \leq \frac{1-\delta^{t_{0}}}{1-\delta} n C^{\prime}
$$

since $\left|m_{i}(\theta)\right| \leq C^{\prime}<\infty .{ }^{7}$ Thus,

$$
\sum_{i=1}^{n} \sum_{t=0}^{t_{0}-1} \delta^{t} \underline{x}_{i}^{T} P^{t} m_{i}-\sum_{i=1}^{n} \sum_{t=0}^{t_{0}-1} \delta^{t} x^{T} P^{t} m_{i}+\sum_{i=1}^{n} \sum_{t=0}^{t_{0}-1} \delta^{t} x^{T} P^{t} v_{i} \geq-\frac{1-\delta^{t_{0}}}{1-\delta} n\left(C+2 C^{\prime}\right) .
$$

Next, we can choose $\epsilon$ small enough so that

$$
\sum_{\theta^{\prime} \in \Theta}\left\{\left(\mu_{\theta^{\prime}}-\epsilon\right)\left(\sum_{i=1}^{n} v_{i, \theta^{\prime}}\right)-2 \epsilon\left(\sum_{i=1}^{n} m_{i, \theta^{\prime}}\right)\right\}>\eta_{0}
$$

for some positive $\eta_{0}>0$. To see this, first note that there exist $\eta_{1}>0$ and $\eta_{2}>0$ such that (i) $\mu_{\theta^{\prime}}>\eta_{1}$ for all $\theta^{\prime} \in \Theta$ by the finiteness of $\Theta$ and (ii) $\sum_{\theta^{\prime} \in \Theta} \sum_{i=1}^{n} v_{i, \theta^{\prime}}=$

7 Observe that $M_{i}(\theta)$ is finite for all $\theta$ since both $W(\theta)$ and $W_{-i}(\theta)$ are finite. Thus, $m_{i}(\theta)$ is finite for all $\theta$. Since $\Theta$ is a finite set, $\left|m_{i}(\theta)\right|$ is bounded by a constant $C^{\prime}<\infty$. 
$\sum_{\theta^{\prime} \in \Theta} \sum_{i=1}^{n} v_{i}\left(\theta_{i}^{\prime}, a^{*}\left(\theta^{\prime}\right)\right)>\eta_{2}$ by the positive surplus assumption. Let $\eta_{0}<\eta_{1} \eta_{2}$ and choose

$$
\epsilon<\min \left\{\eta_{1}, \frac{\eta_{1} \eta_{2}-\eta_{0}}{\eta_{2}+2 n|\Theta| C^{\prime}}\right\}
$$

Then,

$$
\sum_{\theta^{\prime} \in \Theta}\left(\mu_{\theta^{\prime}}-\epsilon\right)\left(\sum_{i=1}^{n} v_{i, \theta^{\prime}}\right)-2 \epsilon \sum_{\theta^{\prime} \in \Theta}\left(\sum_{i=1}^{n} m_{i, \theta^{\prime}}\right)>\left(\eta_{1}-\epsilon\right) \eta_{2}-2 \epsilon n|\Theta| C^{\prime}>\eta_{0}
$$

where $|\Theta|$ is the cardinality of $\Theta$. Note that $\eta_{0}$ is independent of the discount factor $\delta$. Thus, as $\delta \rightarrow 1$, the term $-\left(1-\delta^{t_{0}}\right) n\left(C+2 C^{\prime}\right) /(1-\delta)$ goes to $-t_{0} n\left(C+2 C^{\prime}\right)$ whereas

$$
\frac{\delta^{t_{0}}}{1-\delta} \sum_{\theta^{\prime} \in \Theta}\left\{\left(\mu_{\theta^{\prime}}-\epsilon\right)\left(\sum_{i=1}^{n} v_{i, \theta^{\prime}}\right)-2 \epsilon\left(\sum_{i=1}^{n} m_{i, \theta^{\prime}}\right)\right\}
$$

goes to infinity. Therefore, inequality $(*)$ is satisfied and so ex-ante budget balance is achieved.

This theorem establishes that the dynamic pivot mechanism with lump-sum fees is ex-post efficient, periodic ex-post incentive compatible and individually rational, and exante budget balancing. This was done by showing that inequality $(*)$ is satisfied under appropriate assumptions on the Markov chain and the discount factor: The essence is that the participation constraints of the worst-off types are relaxed when the Markov chain is irreducible and aperiodic and the discount factor is sufficiently large. It is worth mentioning a result of Athey and Segal (2013) at this juncture. They show in their proposition 4 that, with the additional assumption of the independence of transition probabilities across players, budget balance of an efficient policy can be sustained in a perfect Bayesian equilibrium of the decentralized game when the Markov chain has a unique ergodic set and the discount factor is sufficiently large. Compared to their result, we (i) focus on the mechanism design problem per se, (ii) dispense with the independence assumption, and (iii) employ the stronger concept of periodic ex-post incentive compatibility rather than the perfect Bayesian incentive compatibility. Moreover, while they impose the ergodicity 
assumption directly on the efficient policy, we impose the irreducibility and aperiodicity assumption on the transition probability. ${ }^{8}$ On the other hand, their result applies to general efficient policies whereas our result is concerned with the dynamic pivot mechanism.

It is now straightforward to see that budget balance cannot be achieved if the participation constraints of the worst-off types are very tight. One such case may occur when the diverse preference assumption of Bergemann and Välimäki (2010) holds. By part (i) of the diverse preference assumption, for all $i$, there exists $\underline{\theta}_{i} \in \Theta_{i}$ such that player $i$ 's type stays at $\underline{\theta}_{i}$ forever and $v_{i}\left(\underline{\theta}_{i}, a\right)=0$ for all $a \in A$. This implies that $M_{i}\left(\underline{\theta}_{i}, \theta_{-i}\right)=0$ and so $m_{i}\left(\underline{\theta}_{i}, \theta_{-i}\right)=0$ for all $\theta_{-i}$. Thus, the right-hand side of $(*)$ is zero when $\underline{\theta}_{i}$ for all $i$ lies in the support of the initial distribution $x \in \Delta(\Theta)$, and so the inequality cannot be satisfied unless $\sum_{t=0}^{\infty} \delta^{t} x^{T} P^{t} z^{*} \geq 0$.

Note that the diverse preference assumption makes the Markov chain reducible and keeps the payoffs of the worst-off types to zero in this case. Hence, the mechanism cannot charge positive participation fees to cover the budget deficit. Needless to say, budget balance may be achieved for some initial distributions even under the diverse preference assumption. A trivial example can be easily constructed when the initial distribution is concentrated on a type profile, say $\bar{\theta}=\left(\bar{\theta}_{1}, \ldots, \bar{\theta}_{n}\right)$, and players' types stay there forever. More generally, budget balance is possible when $\underline{\theta}_{i}$ 's are not in the support of the initial distribution and these states are entered only with a small probability. ${ }^{9}$

\section{Discussion}

We have shown that the dynamic pivot mechanism can be modified with lump-sum fees so that it is ex-post efficient, periodic ex-post incentive compatible, periodic ex-post individually rational, and ex-ante budget balancing when the Markov chain is irreducible and aperiodic and players are sufficiently patient. We have also shown that budget balance

\footnotetext{
8 Since an efficient policy is stationary with $a^{*}: \Theta \rightarrow A$, the assumptions on the induced Markov chain should ultimately pertain to the transition probability.

9 I thank an anonymous referee for pointing this out.
} 
may not be achieved under the diverse preference assumption.

Observe that Theorem 1 in particular implies that the impossibility result of Myerson and Satterthwaite (1983) for bilateral trading no longer holds in the dynamic setting. Concerning the dynamic bilateral trading problem, Skrzypacz and Toikka (2015) establish the payoff-equivalence property in quite a general setup with multi-dimensional asymmetric information at the initial period, and then characterize a necessary and sufficient inequality condition for the existence of efficient, incentive compatible, individually rational, and budget-balancing mechanisms. ${ }^{10}$ With the inequality condition, they explore how the feasibility of efficient trading depends on (i) the persistence of the Markov process, (ii) the initial private information about process parameter, and (iii) the frequency of interaction.

They use the same mechanism, i.e., the dynamic pivot mechanism, to obtain the characterization result. Note that this is an extension to the dynamic setting of the characterizations of Williams (1999) and Krishna and Perry (2000): These papers first show that, under certain conditions on the primitives and/or the mechanism, any incentive compatible mechanism is payoff equivalent to a Groves mechanism, and then characterize necessary and sufficient conditions using this Groves mechanism. In contrast, we focus on the dynamic pivot mechanism itself and explicitly examine when and how the inequality for budget balance may be satisfied, that is, we prove budget balance of the dynamic pivot mechanism with lump-sum fees under appropriate assumptions on the Markov chain and the discount factor. We want to mention that the payoff equivalence property does not hold when the set $\Theta$ of type profiles is finite, implying that there may exist another efficient, incentive compatible, individually rational, and budget-balancing mechanism even when inequality $(*)$ is not satisfied. On the other hand, it appears to be quite hard to prove a result similar to Theorem 1 when $\Theta$ is not finite.

Lamba (2013) also studies the dynamic bilateral trading. He introduces the concept of interim budget balance, establishes a history-dependent version of the payoff equivalence

10 Their inequality is essentially the same as inequality (*) adapted to bilateral trading. 
property, and characterizes a necessary and sufficient condition for the existence of expost efficient, periodic ex-post incentive compatible and individually rational, and interim budget balancing mechanisms via the collateral dynamic VCG mechanism. Hence, this paper is close in spirit to Skrzypacz and Toikka (2015). ${ }^{11}$

Let $\theta^{t-1}$ be the type profile in period $t-1$, and let $x\left(\theta^{t-1}\right)$ be the distribution of type profile $\theta^{t}$ in period $t$, given $\theta^{t-1}$. In addition, let $x\left(\theta^{t-1}\right)_{i \mid \theta_{i}^{t}}$ be the conditional distribution vector of type profile in period $t$, given $\theta^{t-1}$ and $\theta_{i}^{t}$. Interim budget balance is satisfied if, for all $t$ and $\theta^{t-1}$,

$$
-\sum_{\tau=t}^{\infty} \delta^{\tau-t} x\left(\theta^{t-1}\right)^{T} P^{t} z^{*} \leq \sum_{i=1}^{n} \inf _{\theta_{i}^{t} \in \Theta_{i}\left(x\left(\theta^{t-1}\right)\right)} \sum_{\tau=t}^{\infty} \delta^{\tau-t} x\left(\theta^{t-1}\right)_{i \mid \theta_{i}^{t}}^{T} P^{t} m_{i}
$$

Interim budget balance is obviously stronger than ex-ante budget balance. ${ }^{12}$ On the other hand, observe that, in the (infinite-horizon) stationary environment of the present paper, interim budget balance is implied by ex-ante budget balance if (i) ex-ante budget balance holds for a set of possible initial distributions, denoted by $\Delta_{0}(\Theta) \subseteq \Delta(\Theta)$, and (ii) $x\left(\theta^{t-1}\right) \in \Delta_{0}(\Theta)$ for all $t$ and $\theta^{t-1}$. Take, for example, the case when $\Delta_{0}(\Theta)$ consists of all full-support distributions, and the transition matrix $P$ induces a full-support distribution for any full-support distribution. Another case is when $\Delta_{0}(\Theta)=\Delta(\Theta)$ and so ex-ante budget balance holds for any initial distribution $x \in \Delta(\Theta)$.

We now show that Theorem 1 in fact implies, under the assumptions of that theorem, interim budget balance is achieved for sufficiently large $\delta$. We have $x\left(\theta^{t-1}\right)=x\left(\theta_{t-1}\right)$ by the Markov property, and the set $\bar{\Delta}(\Theta)=\left\{x\left(\theta_{t-1}\right) \mid t=0,1, \ldots\right\}$ of all possible distributions in any period is finite when $\Theta$ is finite. For each member of $\bar{\Delta}(\Theta)$, there exists a critical value of $\delta$ such that ex-ante budget balance is achieved for $\delta$ greater than or equal to this value by Theorem 1 . Let $\bar{\delta}$ be the maximum of these critical values, which is bounded away

11 By the way, both Skrzypacz and Toikka (2015) and Lamba (2013) discuss the second-best mechanism in limited environments.

12 We have $x\left(\theta^{t-1}\right)=x$ when $t=0$. 
from 1 by the finiteness of $\bar{\Delta}(\Theta)$. Thus, interim budget balance is achieved for $\delta \geq \bar{\delta}{ }^{13}$

Finally, note that the theorem was established for the case when the type space is finite and the Markov chain is independent of the actions. Hence, it is an interesting future agenda to see whether this result continues to hold when the type space is countably infinite or even more general, and when the transition kernel depends on the actions.

\section{Acknowledgements}

I thank an advisory editor and anonymous reviewers for many helpful comments and suggestions.

\section{References}

Athey, S. and Segal, I., 2013. An efficient dynamic mechanism. Econometrica 81, 24632485.

Bergemann, D. and Said, M., 2010. Dynamic auctions: A survey. manuscript.

Bergemann, D. and Välimäki, J., 2010. The dynamic pivot mechanism. Econometrica 78, 771-789.

Krishna, V. and Perry, M., 2000. Efficient mechanism design. manuscript.

Lamba, R., 2013. Repeated bargaining: A mechanism design approach. manuscript.

Makowski, L. and Mezzetti, C., 1994. Bayesian and weakly robust first best mechanisms: Characterizations. Journal of Economic Theory 64, 500-519.

Myerson, R. and Satterthwaite, M., 1983. Efficient mechanisms for bilateral trading. Journal of Economic Theory 29, 265-281.

Pavan, A., Segal, I., and Toikka, J., 2014. Dynamic mechanism design: A Myersonian approach. Econometrica 82, 601-653.

Puterman, M., 2005. Markov Decision Processes: Discrete Stochastic Dynamic Programming. John Wiley \& Sons.

13 Obviously, the critical value of $\delta$ may be different from that for ex-ante budget balance. Observe also that this reasoning may not hold when $\Theta$ is not finite. 
Skrzypacz, A. and Toikka, J., 2015. Mechanisms for repeated trade. American Economic Journal: Microeconomics, forthcoming.

Stokey, N., and Lucas, R., 1989. Recursive Methods in Economic Dynamics. Harvard University Press.

Vohra, R., 2012. Dynamic mechanism design. Surveys in Operations Research and Management Science 17, 60-68.

Williams, S., 1999. A characterization of efficient, bayesian incentive compatible mechanisms. Economic Theory 14, 155-180.

Yoon, K., 2001. The modified Vickrey double auction. Journal of Economic Theory 101, $572-584$.

Yoon, K., 2008. The participatory Vickrey-Clarke-Groves mechanism. Journal of Mathematical Economics 44, 324-336. 